\title{
A prospective study of psychiatric disorder and cognitive function in systemic lupus erythematosus
} Elaine M Hay, Alice Huddy, Dawn Black, Patrick Mbaya, Barbara Tomenson,
Robert M Bernstein, P J Lennox Holt, Francis Creed
ARC Epidemiology

Research Unit,

University of

Manchester,

Manchester,

United Kingdom

E M Hay

Manchester Royal

Infirmary,

Manchester,

United Kingdom

Department of

Rheumatology

R M Bernstein

P J Lennox Holt

Department of

Psychiatry

A Huddy

D Black

P Mbaya

B Tomenson

F Creed

Correspondence to:

DrEM Hay,

ARC Epidemiology
Research Unit,

Stopford Building,

Stopford Building,

Oxford Road, 13 9PT,

United Kingdom.

Accepted for publication

9 February 1994

\begin{abstract}
Objectives-To investigate change in psychiatric disorder and change in cognitive function in patients with systemic lupus erythematosus (SLE) assessed on two occasions two years apart.

Methods-A prospective cohort study of 49 patients with SLE using standardised psychiatric and clinical research methods.

Results-The point prevalence of psychiatric disorder $(20 \%$ and $24 \%)$, and of cognitive impairment $(23 \%$ and $18 \%)$, was similar at first and second interview for the whole group. There was, however, considerable change in individual patient's psychiatric status and cognitive function: only $1 / 9$ patients with impairment on two or more cognitive tests at first interview was still impaired at second interview. Change in cognitive function appeared to mirror change in psychiatric status.

Conclusions-These findings suggest that the previously reported high prevalence of cognitive impairment in SLE may be explained by coexisting psychiatric disorder, rather than reflecting subclinical central nervous system (CNS) involvement.
\end{abstract}

(Ann Rheum Dis 1994; 53: 298-303)

A number of studies have reported cognitive impairment, as demonstrated by psychometric testing, with varying frequency in patients with systemic lupus erythematosus (SLE)..$^{1-5}$ Cognitive impairment appears to be more common than many clinicians might expect, particularly amongst patients with no overt evidence of central nervous system (CNS) disorder. Its clinical significance, and implications regarding prognosis, are unclear. The aetiopathogenesis of cognitive impairment in SLE is also not understood. For example, associations between anti-neuronal antibodies and cognitive impairment reported by some workers ${ }^{6}$ have not been confirmed by others, ${ }^{7}$ and most previous studies have found no association between cognitive impairment and clinical or laboratory markers of disease activity, severity, or corticosteroid medication. ${ }^{1-5} 8$
This lack of association between cognitive impairment and immunological or clinical variables casts doubt on suggestions that cognitive impairment reflects subclinical CNS involvement. ${ }^{2}$ Our previous report indicated that impairment of two tests of cognitive function (Verbal Fluency Test (VFT) and Benton Visual Retention Test (BVRT[A]) was significantly associated with psychiatric disorder, but not with systemic disease activity or corticosteroid therapy. ${ }^{1}$ This led us to suggest an alternative explanation for the reported cognitive impairment in SLE, namely that cognitive impairment might result from coexisting psychiatric disorder, which impairs performance on tests of cognitive function by decreased speed, concentration and motivation.

The present study investigates this hypothesis by comparing change in psychiatric disorder over two years with change in cognitive function, to determine whether the resolution of psychiatric disorder is associated with improvement of cognitive function.

\section{Patients and methods}

STUDY DESIGN

This was a two year prospective study.

STUDY POPULATION

The study subjects were derived from a group of 73 patients with SLE, described in detail elsewhere. ' Briefly, they were consecutive outpatient attenders ( 66 patients) or inpatients (seven patients) recruited from the lupus clinic of the Manchester Royal Infirmary Rheumatism Research Centre during the period of April 1988 to December 1989. All patients who attended during the recruitment period were included unless they were over 70 years of age.

Subjects were reviewed approximately two years (range: two years to two years and eight months) after their first interview, using the same standard clinical and psychiatric methods.

\section{OUTCOME MEASURES}

1 Psychiatric diagnosis Psychiatric assessments used the Present State Examination (PSE), performed by two psychiatrists (DB and PB). ${ }^{9}$ In this research interview, each psychiatric 
symptom is rated according to standard definitions. The results were analysed by a computer programme (CATEGO) to give a psychiatric diagnosis according to the ICD-9 (International Classification of Diseases, Revision 9) and an index of definition (ID). Subjects rated as ID 5 or above are considered to have a psychiatric disorder which is severe enough to merit treatment; referred to below as 'cases'. It is somewhat higher than the threshold for diagnosis by the DSM-III (Diagnostic and Statistical Manual of Mental Disorders, 3rd edition).

2 Cognitive function Cognitive function was assessed by the same clinical psychologist (AH) who did the first assessments. She was blind to the psychiatric, clinical and laboratory findings, and to the results of the patients' first assessments. The following tests were performed: Wechsler Adult Intelligence Scale (WAIS) shortened version, ${ }^{10}$ Verbal Fluency Test (VFT), ${ }^{11}$ the Benton Visual Retention Test A (BVRT), ${ }^{12}$ and the Wechsler Memory Scale (WMS). ${ }^{13}$ These cognitive tests have been shown to have good test-retest reliability and to have no learning effect when performed after a time interval greater than six months. ${ }^{14}$ The results of cognitive function tests were analysed in two ways. First, patients were categorised as either normal or impaired according to cut-off values defined elsewhere. ${ }^{1}$ Second, the results of the cognitive tests were analysed as continuous variables. At the baseline assessment mean VFT scores had been lower, and mean BVRT(A) error scores had been higher (indicating poor performance for each test) in the patients with a psychiatric disorder compared with those without. ${ }^{1}$

3 Systemic disease activity and severity Clinical disease activity was measured using the British Isles Lupus Assessment Group (BILAG) index (version 3), applied by a clinical rheumatologist $(\mathrm{EH})$ 'blind' to the psychiatric and psychological assessments. Patients were reviewed at intervals dictated by their clinical state, but at least six monthly. The BILAG index assigns a separate alphabetical score to each of eight organ-based systems, leading to the classification as: ' $A$ ' (active disease thought to require disease modifying treatment), ' $B$ ' (mild reversible activity), ' $C$ ' (stable current involvement), ' $\mathrm{D}$ ' (previous involvement), or ' $E$ ' (system never affected). A total numerical score was calculated using the formula: $A=9$, $\mathrm{B}=4, \mathrm{C}=2, \mathrm{D}=0, \mathrm{E}=0$. The BILAG index has been shown to be a valid and reliable score. ${ }^{15}$

Details of the patients' medical history, with special reference to CNS involvement, were recorded prospectively on a standard chart.

The erythrocyte sedimentation rate (ESR), a complete blood count with differential white cell count, and a serum creatinine level were evaluated by standard techniques. Antinuclear antibody was assessed by indirect immunofluorescence using HEp-2 cells as substrate. ${ }^{16}$ Anti-double stranded DNA (ds-DNA) and anticardiolipin (aCL) antibodies were measured by enzyme-linked immunosorbent assay. ${ }^{17} 18$
4 Social stress and support The Jenkins Social Stress and Support inventory, which was administered by DB and PB, was used to score social stress (from $0=$ no stress to $3=$ severe stress) for occupation, marriage, family, housing, finance and social life. A cumulative score was calculated to measure total stress and lack of social support. ${ }^{19}$

Data analysis Patients were stratified into four groups according to the presence or absence of a psychiatric disorder at initial or second interview (table 1). Changes in clinical features, clinical disease activity, cognitive function and social stress over two years were analysed for the group as a whole, and for the four groups separately using McNemar's test for categorical variables, and Wilcoxon matched pairs signed ranks test for continous variables.

\section{Results}

The study included 47 women and two men, with a median age of 42 years (range 21-70), and a median disease duration of nine years (range 3-42). All met the 1982 revised American Rheumatism Association (ARA) criteria for the classification of SLE. $^{20}$ Complete data for the psychiatric and clinical assessments were available on all 49 patients, but only 38 patients completed both cognitive assessments (see below).

Losses to follow up Of the 73 patients in the original study, there were 24 patients who did not attend for a second psychiatric assessment. The reasons were death (four, including one suicide), leaving the area (three), declined to attend on the second occasion (17): 49 patients were assessed twice. Eleven of these 49 patients did not complete cognitive assessments at both first and second interview: 38 patients had cognitive testing twice.

Patients lost to follow up did not differ significantly from those included in the current study with respect to age, duration of SLE, total social stress and support scores, activity of SLE, degree of cognitive impairment or presence of psychiatric disorder at the first interview. Of the 15 patients with a psychiatric

Table 1 Comparison of psychiatric diagnoses in patients with systemic lupus erythematosus at first and second interviews

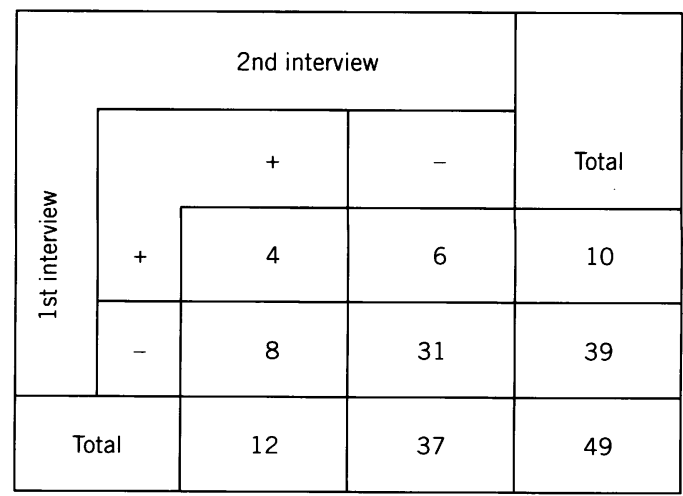

Key: += psychiatric disorder present; $-=$ no psychiatric disorder.

McMemar's test for change $\mathrm{X}_{1}^{2}=0.07$ (NS). 
Table 2 Numbers of patients impaired for each of five cognitive tests: first interview compared with second interview

\begin{tabular}{lllllll}
\hline & $N-I$ & $N-N$ & $I-I$ & $I-N$ & $X_{I}^{2}$ & $p$ Value \\
\hline VFT & 6 & 28 & 2 & 2 & $2 \cdot 0$ & NS \\
BVRT[A] number correct & 2 & 30 & 1 & 5 & $1 \cdot 3$ & NS \\
BVRT[A] number errors & 6 & 23 & 5 & 3 & $1 \cdot 0$ & NS \\
WMS & 4 & 28 & 0 & 6 & $0 \cdot 4$ & NS \\
WAIS & 4 & 26 & 0 & 5 & $0 \cdot 4$ & NS \\
Any 1 test & 7 & 12 & 11 & 8 & $0 \cdot 1$ & NS \\
2 or more tests & 6 & 23 & 1 & 8 & $0 \cdot 3$ &
\end{tabular}

Key: N-I = Not impaired at first interview, impaired at second interview; $\mathrm{N}-\mathrm{N}=$ not impaired at either interview; $\mathrm{I}-\mathrm{I}=$ impaired at both interviews; $\mathrm{I}-\mathrm{N}=$ impaired at first interview, not impaired at second interview.

Significance values calculated using McNemar's test of change.

disorder at the first interview, 10 were followed up $(67 \%)$, compared with 39 of 56 without an initial psychiatric disorder $(70 \%)$.

Four patients died during the study period, including one suicide- $-\mathrm{a}$ male with profound cognitive impairment, recent stroke and depression. The causes of death in the other patients were: stroke (one patient who also had depression and cognitive impairment), and multi-system SLE with predominantly renal (one patient) and cardiovascular (one patient) involvement. These last two patients did not have a psychiatric disorder or cognitive impairment at the initial assessment. The four patients who died had a higher prevalence of clinical manifestations at the initial assessment, indicating more severe SLE.

Psychiatric disorder Psychiatric disorder (ID > 5) was present in $12 / 49(24.5 \%)$ patients at the second interview (eight depression and four anxiety) compared with 10/49 (20\%) at the first interview. Five further patients with psychiatric disorder at first interview were lost to follow up (two had died, including one suicide, two had moved away and one declined to attend).

The numbers of patients with psychiatric disorder at initial or follow up interview are shown in table 1. Although the proportions of patients with psychiatric disorder at each assessment were similar, there was considerable change over time in the psychiatric status of individual patients. Thus psychiatric disorder resolved over two years in six patients, developed for the first time in eight patients, and persisted in only four patients.

Tables 3 Comparison of variables at first and second interview

a Continuous variables

\begin{tabular}{lccc}
\hline & First interview & Second interview & $p$ Value \\
\hline Clinical features $^{\star}$ & $2(1-3)$ & $5(4-6)$ & $<0 \cdot 001$ \\
Prednisolone dose (mg) & $4(0-8)$ & $2(1-3)$ & NS \\
ESR (mm/hr) & $19(8-43)$ & $18(7-41)$ & NS \\
Total stress score & $1(0-3)$ & $2(1-3)$ & $<0 \cdot 01$ \\
Total 'lack of support' score & $0(0-1)$ & $2(0-5)$ & $<0.05$ \\
\hline
\end{tabular}

Table shows median and interquartile range.

$\mathrm{P}$ values calculated using Wilcoxon matched pairs signed ranks test.

$\star$ Prevalence of malar rash, photosensitivity, discoid rash, ulcers, arthritis, serositis, CNS involvement, renal involvement.

b Categorical variables

\begin{tabular}{lccclcl}
\hline & Neg/Pos & Negative & Positive & Pos/Neg & $X_{I}^{2}$ & $p$ Value \\
\hline ANA (titre >100) & 10 & 3 & 35 & 0 & $10 \cdot 0$ & $0 \cdot 002$ \\
Anti dsDNA (titre >15 u/l) & 4 & 35 & 5 & 4 & 0 & NS \\
aCL (titre >15 u/l) & 2 & 42 & 2 & 2 & 0 & NS \\
BILAG 'A' in any system & 8 & 37 & 0 & 4 & $1 \cdot 3$ & NS \\
\hline
\end{tabular}

Key: Neg/pos = negative at first interview, positive at second; negative $=$ at both interviews; positive = at both interviews; pos $/ \mathrm{neg}=$ positive at first, negative at second interview positive = at both interviews; pos/neg = positive at first
P values calculated using McNemar's test of change.
Cognitive function Cognitive impairment on one or more test was present in $18 / 38(47 \%)$ : $11(29 \%)$ were impaired on a single test, and $7(18 \%)$ on two or more tests. The numbers of patients impaired at first and second interviews on each of the five cognitive tests are shown in table 2 . Only $1 / 9$ patients with impairment on two or more tests at first interview was still impaired at the second interview. Six patients showed cognitive impairment at the second interview only.

Systemic disease and severity and activity The prevalence of clinical features (that is, malar rash, photosensitivity, discoid rash, mucocutaneous ulcers, arthritis, serositis, CNS involvement and renal involvement) increased significantly over two years (table 3a). Active SLE (defined as BILAG ' $A$ ' in any system) was present in $8 / 49(16 \%)$ patients at the second interview compared with $4 / 49(8 \%)$ at the first interview (NS) (table 3b). No patients had active SLE at both assessments. A further 11 $(22 \%)$ patients scored BILAG 'A' at some time during the two year follow up. Five patients had an episode of active CNS-SLE during the study (stroke in three patients, epilepsy in one and cranial nerve palsy in one), but none was active at the second psychiatric interview.

Median ESR and prednisolone dose were similar at first and second interview (table 3a). Similar proportions of patients had elevated titres of anti-dsDNA and aCL antibodies at the first and second interview (table $3 \mathrm{~b}$ ).

Social stress and lack of support scores There was a significant increase in total stress score and lack of support score over two years (table 3a).

ASSOCIATIONS BETWEEN CHANGE IN PSYCHIATRIC DISORDER AND CHANGE IN OTHER VARIABLES

For the remaining analyses patients were divided into four subgroups, according to the presence or absence of psychiatric disorder at the first and second interviews, as shown in table 1 .

Cognitive function At the second interview, mean VFT and BVRT[A] scores showed improvement (compared with the first interview) in patients whose psychiatric disorder had resolved ('case, non-case'), but did not improve in the four patients with persisting psychiatric disorder ('case, case') (fig 1, 2). Conversely, mean VFT and BVRT[A] error scores deteriorated in patients with a new psychiatric disorder ('non-case, case'). Mean scores did not change significantly over two years in patients free from psychiatric disorder at both interviews ('non-case, non-case').

This pattern of change in cognitive function was also shown for the BVRT (A) 'number correct' and the WMS tests but not for the WAIS abnormality scores, which did not change significantly over two years (data not shown).

Systemic disease severity and activity The prevalence of clinical manifestations increased for all four subgroups of patients, irrespective 


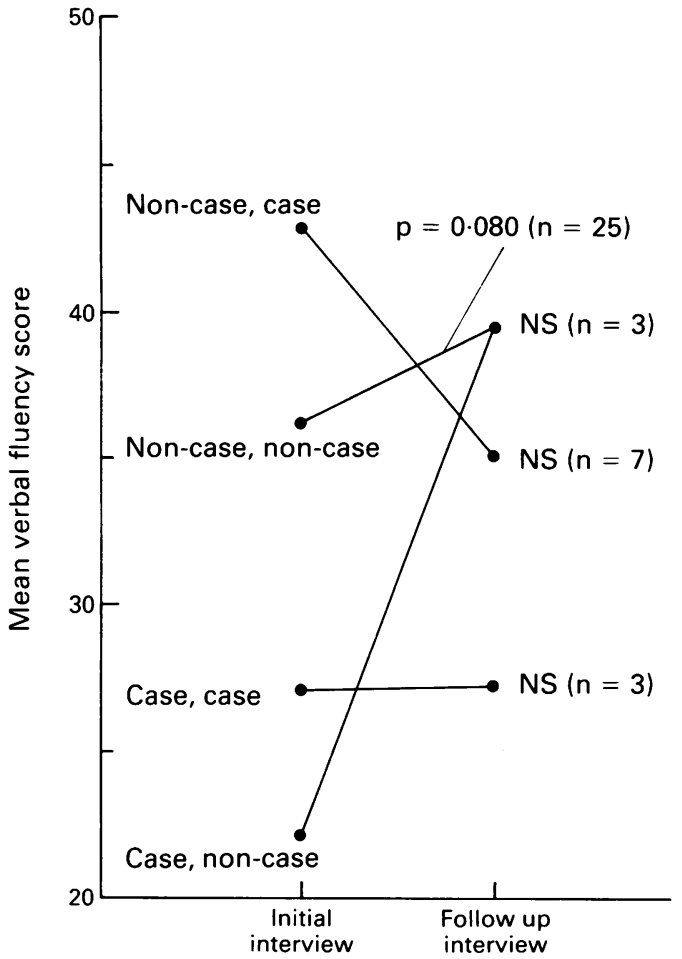

Figure 1 Mean Verbal Fluency Scores for each subgroup at first and second interview. (Low score indicates impairment). . Case, case $=$ psychiatric disorder present at both first and second interview; case, non-case = psychiatric disorder present at first interview but not at second; noncase, non-case $=$ no psychiatric disorder at first or second interview; case, non-case = psychiatric disorder present at second interview, but not at first.

of the presence of psychiatric disorder (fig 3). Disease activity (BILAG score and laboratory data) was also similar for the four groups (data not shown).

Social stress and lack of support scores Total stress scores increased significantly in the eight

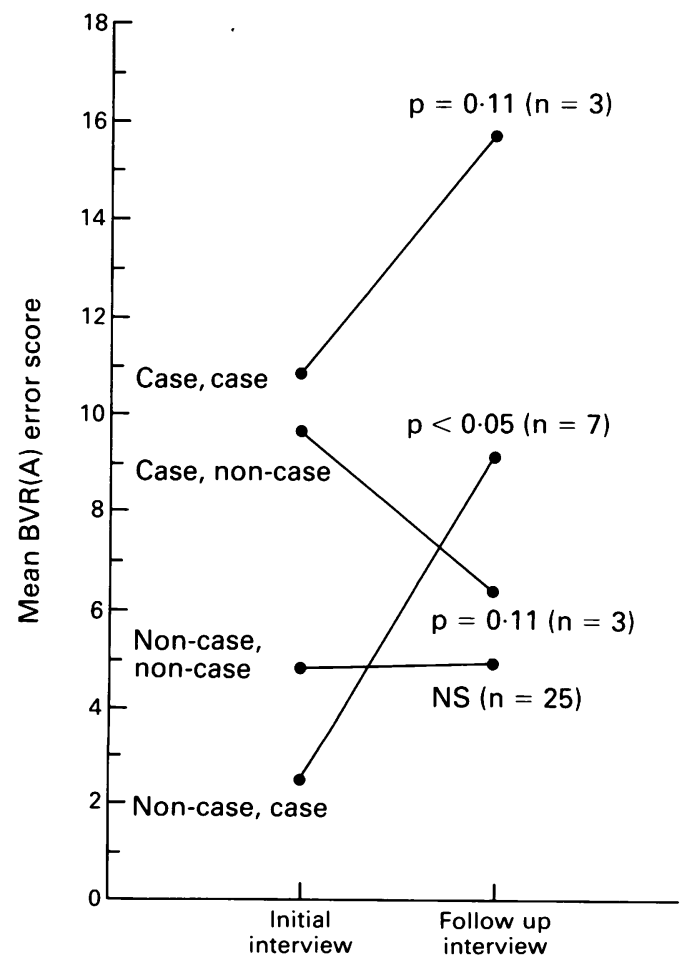

Figure 2 Mean Benton Visual Retention Test $(A)-$ 'number of errors' score for each subgroup at first and second interview (High score indicates impairment) (For Key see fig 1).

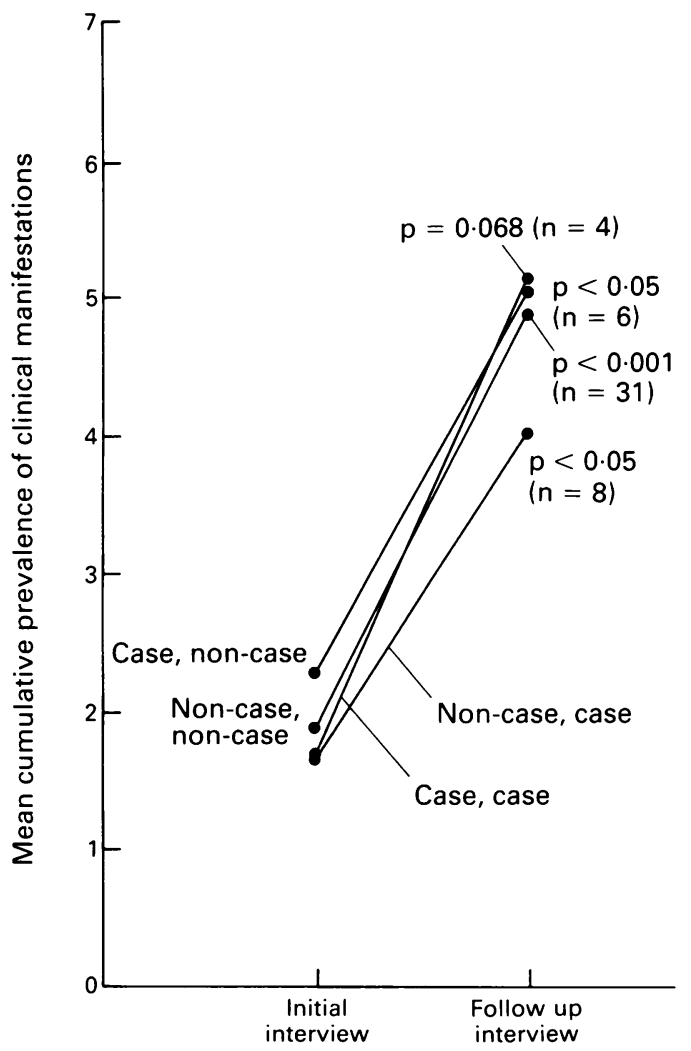

Figure 3 Mean prevalence of clinical manifestations ${ }^{*}$ for each subgroup at first and second interview. (For Key see fig 1).

patients with a new psychiatric disorder ('noncase, case') and, to a lesser extent, in those without a psychiatric disorder at either interview ('non-case, non-case') (fig 4). Patients whose psychiatric disorder resolved ('case, non-case') showed a slight decrease in total stress scores. The results for the 'lack of support' scores showed similar changes (data not shown).

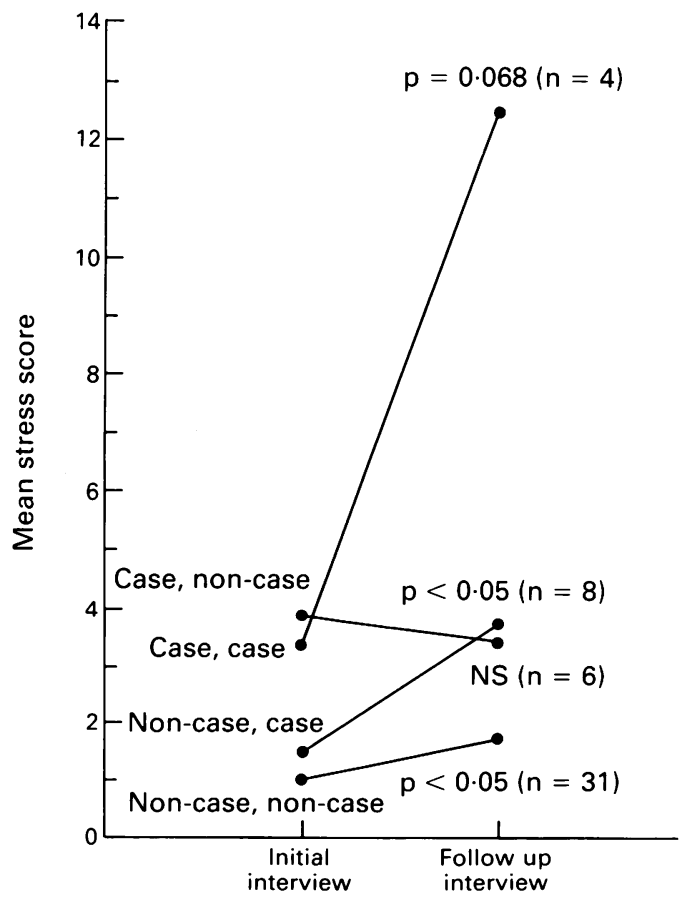

Figure 4 Mean stress score for each subgroup at first and second interview. (For Key see fig 1). 


\section{Discussion}

We have demonstrated considerable change in the psychiatric and cognitive status of patients with SLE over two years. Change in cognitive function appeared to mirror change in psychiatric status.

Subclinical CNS involvement in SLE, which may lead to overt cerebral disorder such as dementia, stroke or epilepsy, is an extremely worrying possibility for patients and clinicians. We previously reported a point prevalance of cognitive impairment of $26 \%,{ }^{1}$ which is similar to the $21 \%$ reported by Hanly et $a l^{8}$ in another cross-sectional study, although much lower than that found by other workers who employed more detailed tests, and used a lower threshold for defining abnormality. ${ }^{2}$ The retrospective or cross-sectional design of most previous studies, ${ }^{1-5}$ however, has meant they have not been able to investigate change in cognitive impairment over time to establish whether it is always a progressive, relentless process. Furthermore, they have not established whether poor performance on cognitive testing performed at a single point in time predicts future CNS events.

In this prospective study, we found no evidence of progressive cognitive impairment amongst patients who were assessed at two points, two years apart. Indeed, most patients who had cognitive impairment at the first assessment had improved by the time of the second assessment: only one of the 10 patients with cognitive impairment on two or more tests at the first interview was still impaired at the second interview. Improvement in cognitive function was noted even amongst some of the patients who had stroke or epilepsy recorded at first interview, although this observation may be confounded by the presence of psychiatric disorder in $7 / 16$ of such patients. None of the five patients who developed a new CNS disorder (stroke in three patients, epilepsy in one and cranial nerve palsy in one) during follow up had been shown to have cognitive impairment on any test at the first interview. Hence, routine cognitive testing, which is time consuming and expensive, would appear unhelpful for identifying patients at future risk of CNS disorder.

We demonstrated that cognitive scores of individual patients, for all tests except the WAIS, improved dramatically amongst patients whose psychiatric disorder (present at the first interview) resolved over two years. By contrast, cognitive scores deteriorated in those patients with either ongoing or new psychiatric disorder. Patients who were free from either psychiatric disorder or overt CNS disorder during the study showed no change in cognitive function over two years. Our findings suggest that cognitive impairment in SLE can be partly explained by coexisting psychiatric disorder which decreases speed, concentration and motivation. Hanly et $a l^{21}$, followed 72 patients with SLE for one year and also found that cognitive impairment resolved in the majority, but no comparison between cognitive impairment and psychiatric status was made in that study.
As expected, there was a significant increase in the prevalence of clinical features over two years, indicating progression of the disease process. There was a lack of association between change in psychiatric status and change in disease activity, severity, or corticosteroid medication. By contrast, there were highly significant associations between change in psychiatric disorder and change in social stress and lack of support scores, thus supporting previous reports proposing that psychiatric disorder in SLE is more closely associated with concurrent psychological stresses than with the course of a painful debilitating illness. ${ }^{122}$

The principal limitation of this study was the reduced number of patients who were able to complete psychiatric and cognitive assessments on both occasions. Forty nine patients from the original cohort of 73 completed both psychiatric assessments but, of these, only 38 completed both cognitive assessments. Most of the losses to follow up declined a second psychological assessment. Those reviewed were similar to the patients lost to follow up for all variables recorded, hence our findings can be generalised to other similar groups of lupus patients. To avoid potential bias resulting from patients lost to follow up we used paired analyses restricted to the 49 patients on whom we had complete psychiatric and clinical data, or the 38 on whom we had complete cognitive data where appropriate. Despite small numbers our study yielded clear results which can be considered valid.

Psychiatric disorder is common in SLE and is independently associated with social stress $^{122}$ and two indicators of cognitive function. ${ }^{1}$ The present study extends these findings by showing that cognitive function returns to normal when psychiatric disorder resolves, in patients with SLE followed for two years. They indicate, once again, the importance of detecting and treating psychiatric disorder in patients with SLE.

The research was supported by a grant from North West Regional Health Authority. Dr Hay was funded by an ARC Junior Research Fellowship.

1 Hay E M, Black D, Huddy A, et al. Psychiatric disorder and cognitive impairment in systemic lupus erythematosus. Arthritis Rheum 1990; 35: 411-6.

2 Carbotte R M, Denburg S D, Carbotte R M, Singal D P, Denburg J A. Prevalence of cognitive impairment in SLE. 7 Nerv Ment Dis 1986; 174: 357-64.

3 Hanly J G, Fisk J D, Sherwood G, Jones E, Verrier Jones J, Eastwood B. Cognitive impairment in patients with systemic lupus erythematosus. F Rheumatol 1992; 19 $562-7$.

4 Ginsburg K S, Wright E A, Larson M G, et al. A controlled study of the prevalence of cognitive dysfunction in randomly selected patients with systemic lupus erythematosus. Arthritis Rheum 1992; 35: 776-82.

5 Wekking E M, Nossent J C, van Dam A P, Swaak A J J G. Cognitive and emotional disturbances in systemic lupus erythematosus. Psychother Psychosom 1991; 55: 126-31.

6 erythematosus. Psychother Psychosom 1991; 55: 126-31. Denburg J A. Serum lymphocytotoxic antibodies and neurocognitive function in SLE. Ann Rheum Dis 1990; 49: 249-25.

7 Papero P H, Bluestein H G, White P, Lipnick R N. Neuropsychological deficits and antineuronal antibodies in paediatric systemic lupus erythematosus. Clin Exp in paediatric systemic lup

8 Hanly J G, Walsh N M, Fisk J D, et al. Cognitive impairment and autoantibodies in systemic lupus erythematosus. and autoantibodies in systemic

9 Dean C, Surtees P, Sashidharan S. Comparisons of research diagnostic systems in an Edinburgh community sample. Br f Psychiat 1983; 142: 247-56. 
10 Wechsler Adult Intelligence Scale. Windsor: NFER, Nelson Publishing Company. 1971.

11 Edgar $M$. Verbal fluency as a function of verbal intelligence and in relation to different types of cerebral pathology. and in relation to different types of
$B r \mathcal{F}$ Clin Psychology 1984; 23: 53-7.

12 Benton A. Benton Visual Retention Test: Clinical and Experimental Applications. New York: The Psychological Corporation, 1974.

13 Wechsler Memory Scale. New York: The Psychological Corporation, 1971.

14 Lezak M D. Neuropsychological assessment. Oxford: Oxford University Press, 1983.

15 Hay E M, Bacon P A, Gordon C, et al. The BILAG index: a reliable and valid instrument for measuring clinical disease activity in systemic lupus erythematosus. Quart $\mathcal{f}$ Med 1993; 86: 447-58.

16 Bernstein R M, Steigerwald J C, Tan E M. Association of antinuclear and antinucleolar antibodies in progressive antinuclear and antinucleolar antibodies in progressive
systemic sclerosis. Clin Exp Immunol 1982; 48: 43-51.
17 Klotz J L, Minami R M, Teplitz R L. An enzyme linked immunosorbent assay for antibodies to native and denatured DNA. F Immunol Methods 1979; 29: 155-65.

18 Snowden N, Wilson P, Pumphrey R S H. Anticardiolipin antibodies in systemic lupus erythematosus. Ann Rheum antibodies in system $1989 ; 48:$ 80-6.

19 Jenkins R, Mann A H, Belsey E. The background, design and use of a short interview to assess social stress and support in research and clinical settings. Soc Sci Med 1981; 15: 195-203.

20 Tan E M, Cohen A S, Freis J F, et al. The 1982 revised criteria for the classification of systemic lupus erythematosus. Arthritis Rheum 1982; 25: 1271-7.

21 Hanly J G, Fisk J D, Sherwood G, Eastwood B. A prospective analysis of cognitive dysfunction in systemic lupus erythematosus. Arthritis Rheum 1992; 35: S60.

$22 \mathrm{Lim}$ L, Ron M A, Ormerod I E C, et al. Psychiatric and neurological manifestations in systemic lupus neurological manifestations in system.
erythematosus. Quart $f$ Med 1988; 66: $27-38$. 